\begin{tabular}{cc}
\hline International Journal of Engineering \& Technology, $7(2.24)(2018) 42-46$ \\
SPC \\
Website: ww. wsiencepubco.com/index.php/IJET \\
Research paper
\end{tabular}

\title{
Vision based Office Automation and Security System using Machine Learning and Internet of Things
}

\author{
Amber Goel*, Apaar Khurana, Pranav Sehgal, K. Suganthi \\ Department of Electronics and Communication, SRM Institute of Science and Technology, Chennai \\ *Corresponding author E-mail: ambergl2014@gmail.com
}

\begin{abstract}
The paper focuses on two areas, automation and security. Raspberry Pi is the heart of the project and it is fuelled by Machine Learning Algorithms using Open CV and Internet of Things. Face recognition uses Linear Binary Pattern and if an unknown person uses their workstation, a message will be sent to the respective person with the photo of the person who uses the workstation. Face recognition is also being used for uploading attendance and switching ON and OFF appliances automatically. During un-official hours, A Human Detection algorithm is being used to detect the human presence. If an unknown person enters the office, a photo of the person will be taken and sent to the authorities. This technology is a combination of Computer Vision, Machine learning and Internet of things, that serves to be an efficient tool for both automation and security.
\end{abstract}

Keywords: Facial Recognition; Histogram of Oriented Gradients; Human Detection; Linear Binary Pattern; Raspberry Pi; Support Vector Machine;.

\section{Introduction}

Computer vision based human detection in surveillance systems provides assistance to public and private organizations like schools, colleges etc. for monitoring purposes and prevention of wrongdoings. Detection of humans in office premises during nonoffice hours for security purposes. The detection and recognition of human faces is being used for the purpose of employee attendance and to gain access to cabin power access. Private access to individual workstation with detection of outside human intervention and remote shutdown capability has been designed for this project.

\section{Methodology}

\subsection{Algorithm for Human Detection}

An image of the object is captured and a Histogram of oriented gradients is formed on the basis of features. A support vector machine is used to classify the object as human and the output is displayed as a picture of the person.

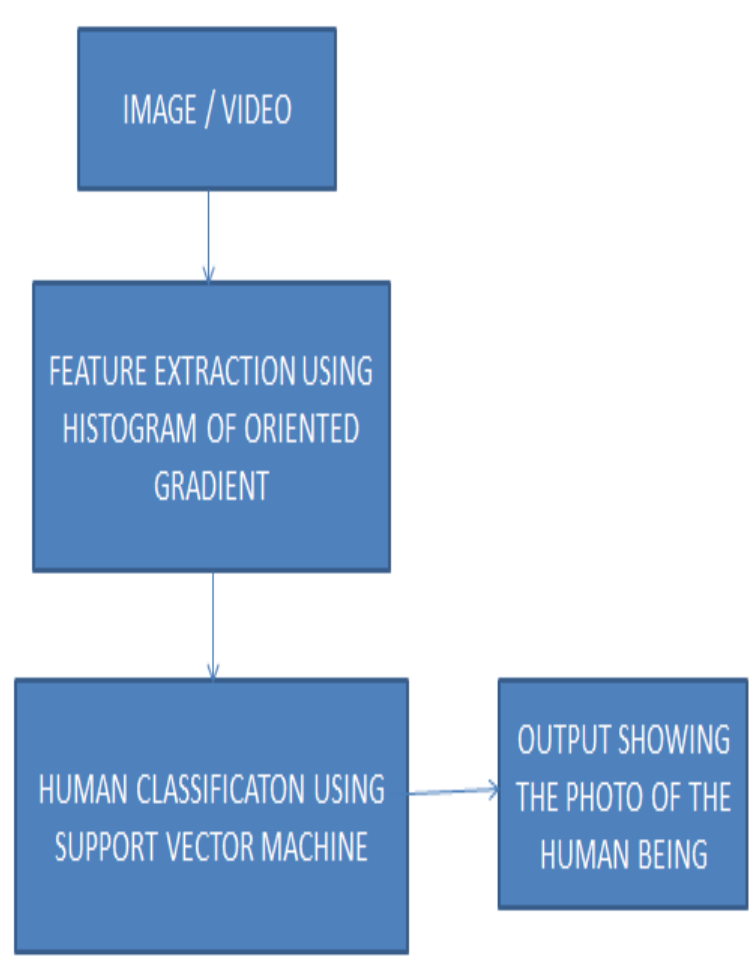

Fig. 1: Algorithm for Human Detection 


\subsection{Algorithm for Face Recognition}

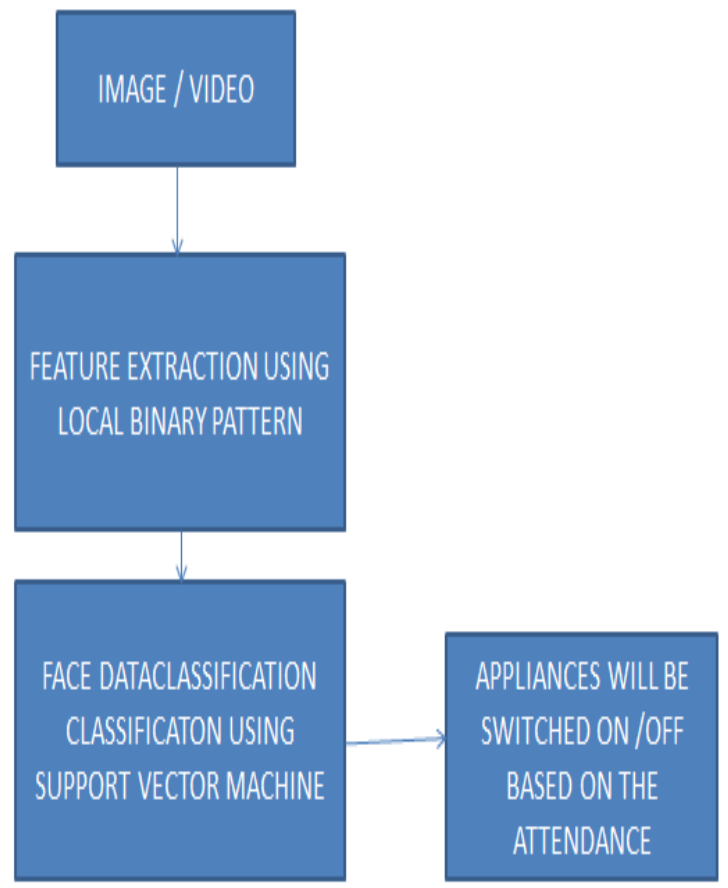

Fig. 2: Algorithm for Face Recognition

\subsection{Local Binary Pattern}

Local Binary Pattern is a visual descriptor needed for classification in the field of computer vision. It is realised by dividing the examined window into cells. For every pixel in a cell, the pixel is compared to all of its nearby cell (on its left-top, left-middle, left-bottom, right-top). If the centre pixel's value is greater than it's neighbour's value, we assign "0". Else, we write "1". This gives an 8-digit binary number (which is converted into decimal number for further comparision).

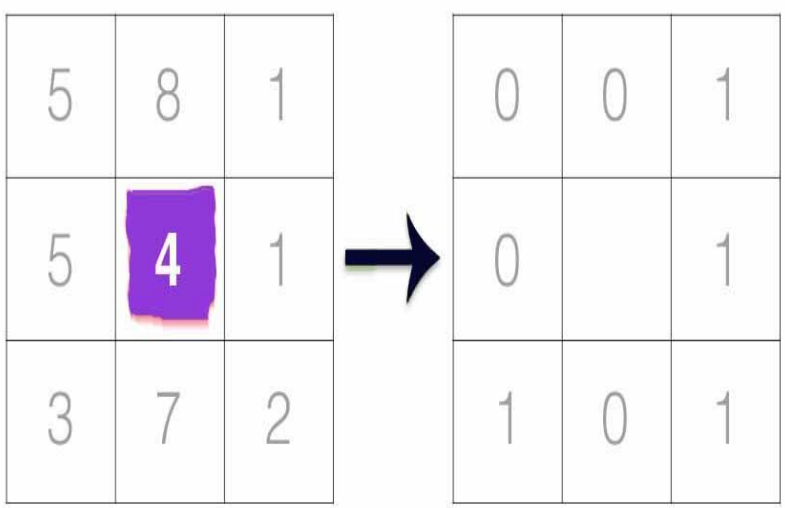

Fig. 3 : Local Binary Pattern

\subsection{Histogram of Oriented Gradients}

The feature descriptor being used in our project uses computer vision and is known as a histogram of oriented gradients. The system counts incidences of gradient orientation in contained portions of an image. The object's appearance can be defined by the intensity of change in light in an image. Cells are created of the image, they are analysed and a histogram of oriented gradients is formed.

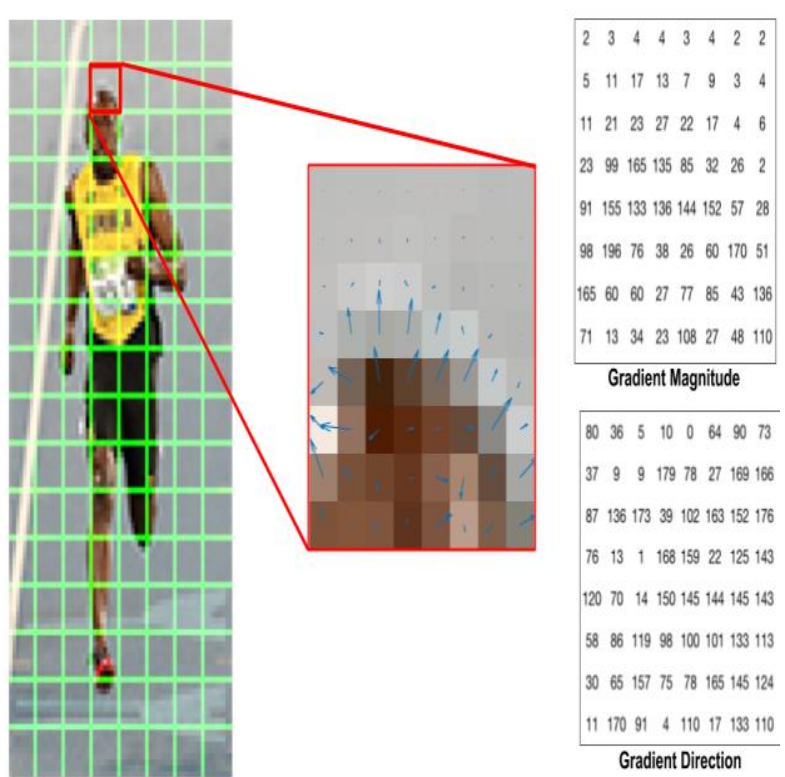

Fig. 4: Histogram Oriented Gradient

\subsection{Support Vector Machine}

Support Vector Machine algorithms in machine learning analyses classification data and does regression analysis. Among training examples, it builds a model which separates items into different categories which puts it in the category of a binary filter. It divides categories in hyperplanes which can be used for detection.

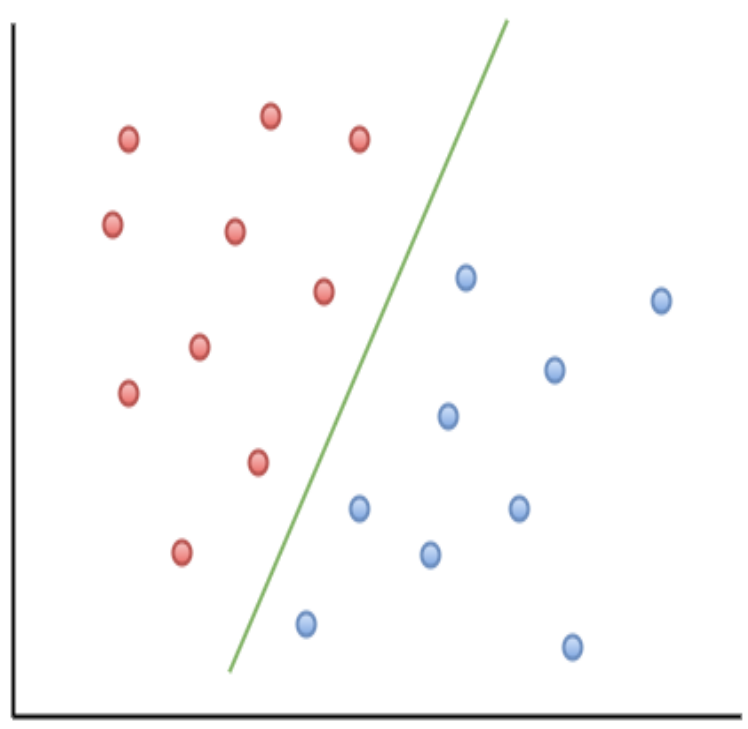

Fig. 5: Support Vector Machine

An image of the face is captured and its features are extracted using Local Binary Pattern. The data is classified using support vector machine and attendance and cabin power access is gained if the face matches the database.

\section{System Design}

\subsection{Hardware Design for Human Detection}

The camera, wi-fi and the relay connected to the alarm are being controlled by the raspberry pi. 


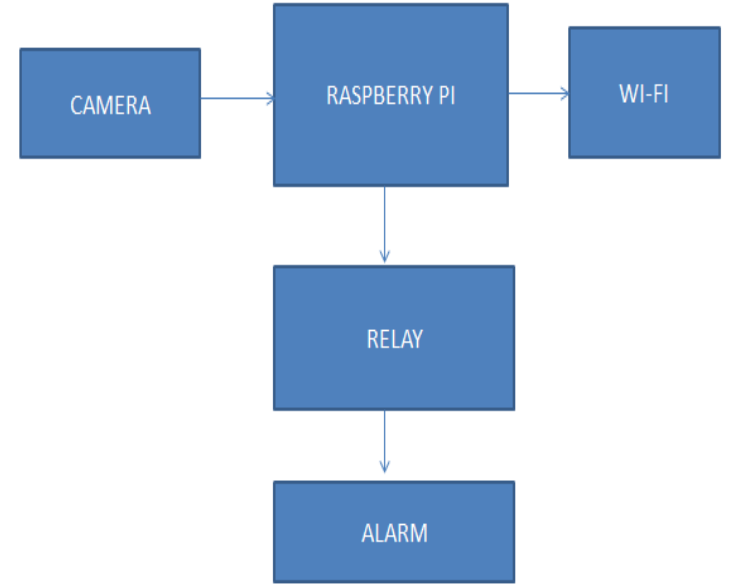

Fig. 6: Hardware Design for Human Detection

The picture captured if a third party tries to enter the office in nonoffice hours, is then uploaded on the Amazon AWS Server and link is sent to the security officer using Twilio SMS.

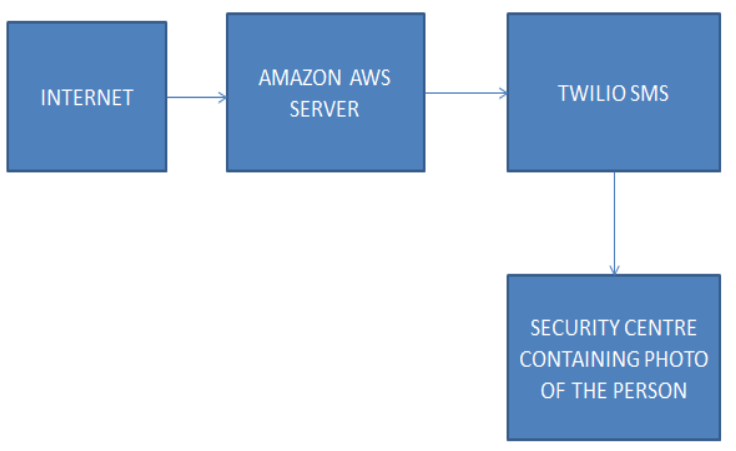

Fig. 7: Human Detection Monitoring

\subsection{Hardware Design for Face Recognition and Attendance System}

An image of the face is captured and its features are extracted using Local Binary Pattern. The data is classified using support vector machine and attendance and cabin power access is gained if the face matches the database.

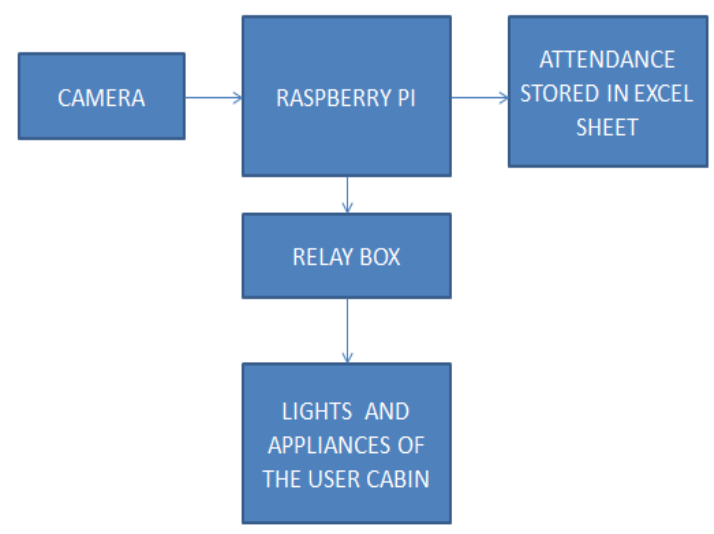

Fig. 8: Face recognition based attendance system

\subsection{Hardware Design for Workstation Security}

The picture captured if a third party tries to access the workstation is then uploaded on the Amazon AWS Server and link is sent to the Cabin User using twilio SMS.

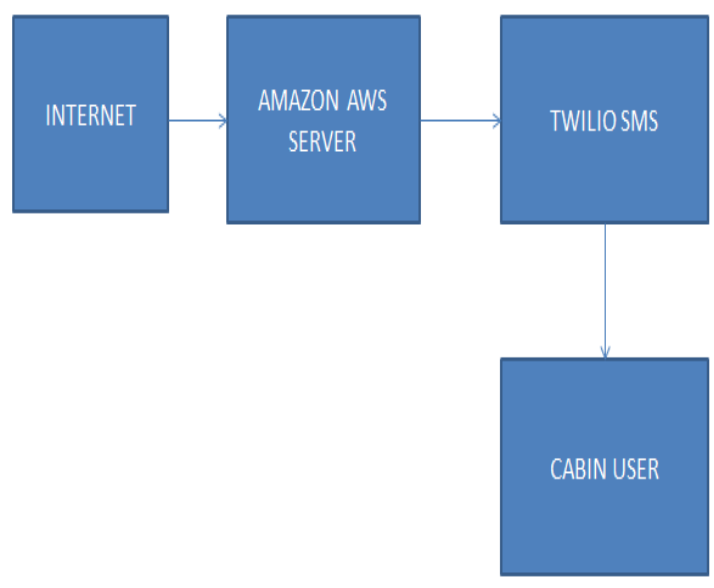

Fig. 9: Workstation Security

\section{Result}

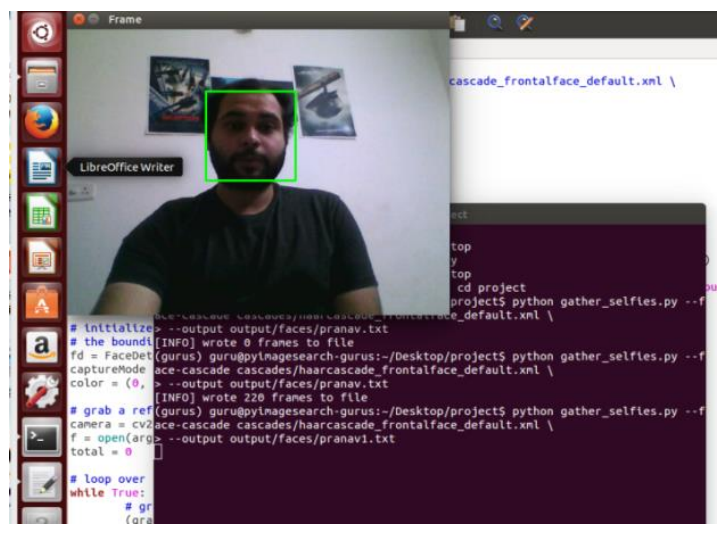

Fig. 10: Face Detection

The face is detected and is highlighted by a green square. If the face is the correct one then the letter ' $C$ ' is typed to start taking clicks to add in the database. To stop adding frames the letter ' $Q$ ' needs to be clicked. These images are saved with the name of the person.

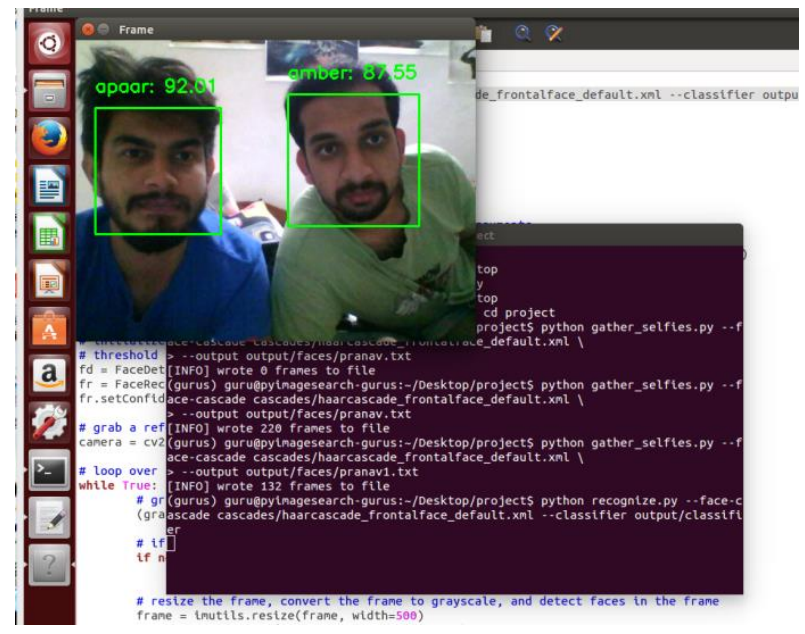

Fig. 11: Face Recognition - Multiple Faces

During recognition stage, the new picture faces are compared with the ones in the database. The comparison is done with help of Support Vector Machine. The attendance for the matched faces is then uploaded on the excel sheet. 


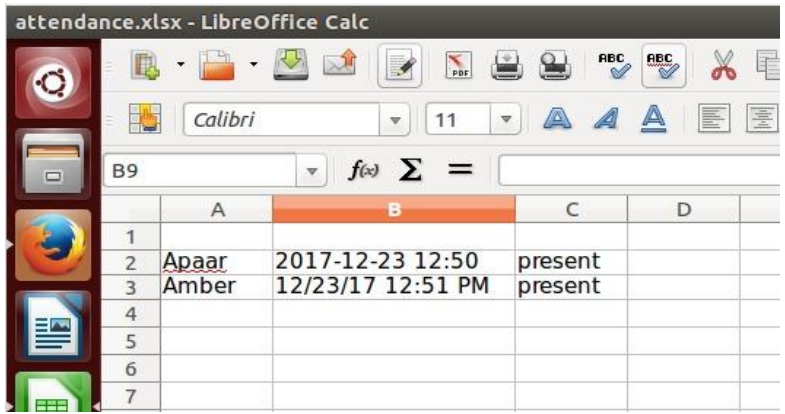

Fig. 12: Attendance uploaded on Excel

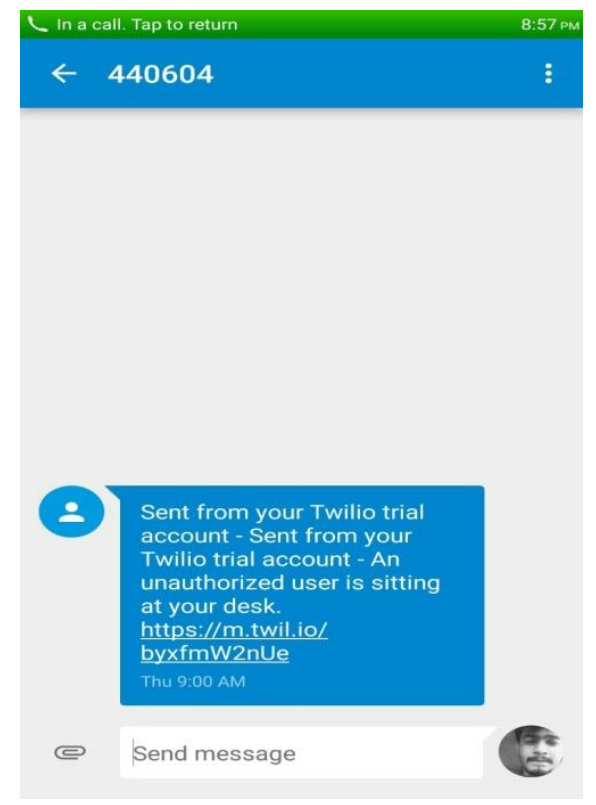

Fig. 13: SMS Received on unauthorized user

This is the result for the second objective, if an authorised person is sitting on a workstation then his photo is uploaded on the AWS server and the link of the photo is sent to the workstation user.

\section{$4 . \mathbf{A}$}

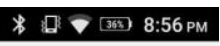

a i-external-1.amazonaws.com/r 4 (7)

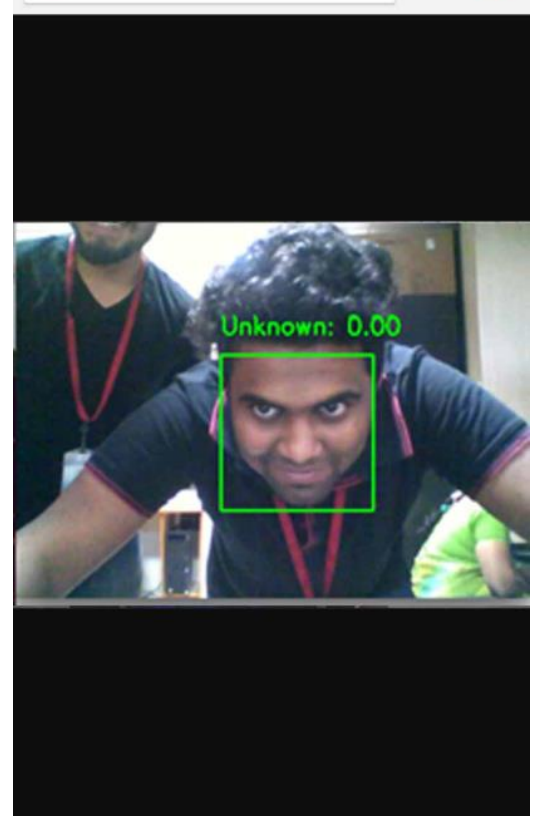

Fig. 13: AWS Image of unauthorized user

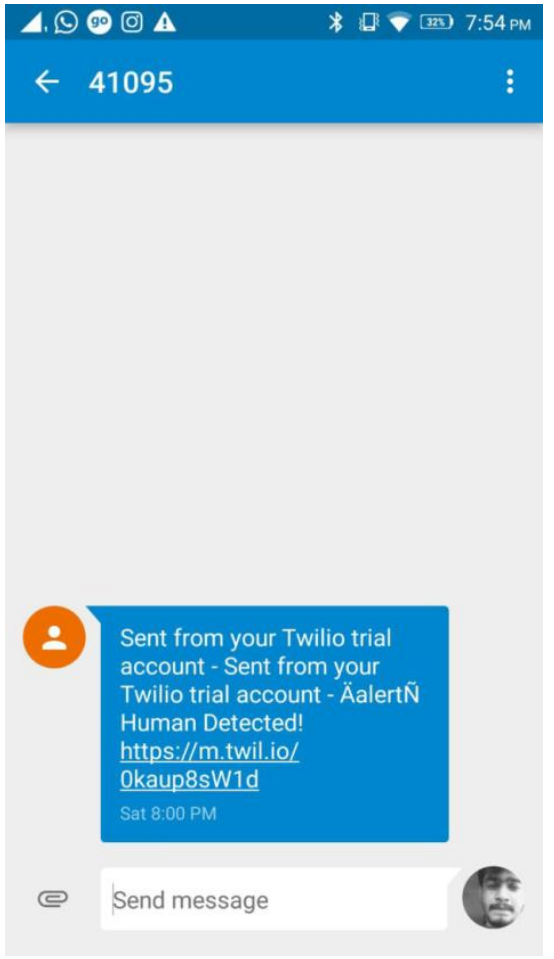

Fig. 14: SMS Received on Human Detection

The image indicates the SMS received on presence of person in the office during non-office hours. The message includes an alert and the link of the image which is uploaded on the AWS server. This message is sent to the security officer.

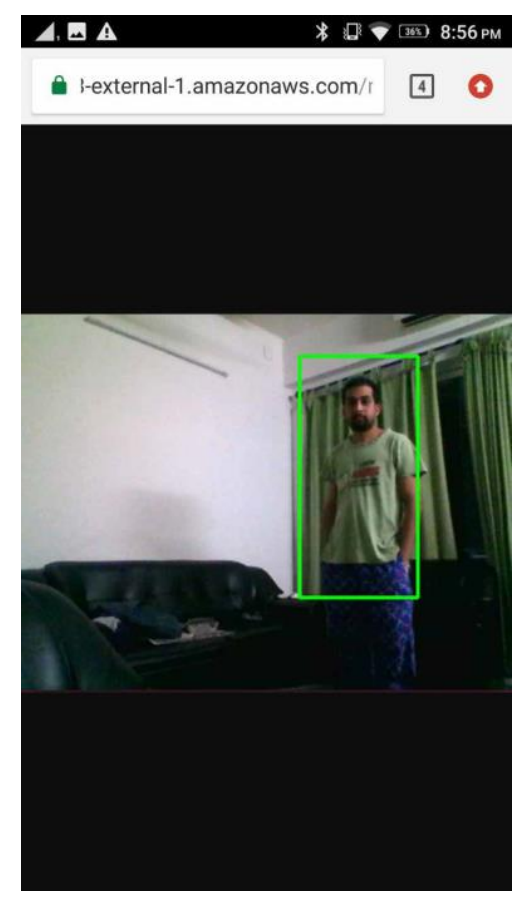

Fig. 15 : Human Detection

\section{Conclusion}

This paper successfully demonstrates 3 objectives: facial recognition and human detection and workstation security. The paper ended with accurate and cost effective solution for office automation and security. Although better results can be gathered with better equipment, the concept and its implementation show that it successfully detects documented faces, uploading 
attendance and controlling appliances, provides individual workstation security and detects the presence of humans when operational.

\section{Future Work}

Many future developments can be made using facial recognition technology like at airports and railway stations to forego security check. Mood detection can be used to chart down employee reviews and satisfaction levels. Human detection technology is getting better and better in the autonomous vehicle market and continues to get better till a fully autonomous level is reached.

\section{Acknowledgement}

We would like to express our sincere gratitude to the staff of Department of Electronics and Communication, SRM Institute of Science and Technology, Chennai for their encouragement and support.

\section{References}

[1] Shubham Mathur, Balaji Subramanian, Sanyam Jain, Kajal Choudhary "Human Detector and Counter Using Raspberry P Microcontroller", Human Detector and Counter Using Raspberry Pi Microcontroller 2017

[2] E.Varadharajan,R.Dharani, S.Jeevitha, B.Kavinmathi, S.Hemalatha "Automatic Attendance Management System Face Detection", International Conference on Green Engineering and Technologies (IC-GET) 2017

[3] Ishita Gupta, Varsha Patil, Chaitali Kadam, Shreya Dumbre “ Face Detection and Recognition using Raspberry Pi “ IEEE International WIE Conference on Electrical and Computer Engineering (WIECON-ECE) 2016

[4] Bhaumik Vaidya, Ankit Patel, Anand Panchal "Smart home automation with a unique door monitoring system for old age people using Python, OpenCV, Android and Raspberry pi ", International Conference on Intelligent Computing and Control Systems (ICICCS) 2017

[5] S.V. Manikanthan , T. Padmapriya "An enhanced distributed evolved node-b architecture in 5G tele-communications network" International Journal of Engineering \& Technology (UAE), Vol 7 Issues No (2.8) (2018) 248-254.March2018

[6] S.V. Manikanthan, T. Padmapriya, Relay Based Architecture For Energy Perceptive For Mobile Adhoc Networks, Advances and Applications in Mathematical Sciences, Volume 17, Issue 1, November 2017, Pages 165-179

[7] S.V.Manikanthan and T.Padmapriya "Recent Trends In M2m Communications In 4g Networks And Evolution Towards 5g", International Journal of Pure and Applied Mathematics, ISSN NO:1314-3395, Vol-115, Issue -8, Sep 2017. 